\title{
A representative particle approach to coagulation and fragmentation of dust aggregates and fluid droplets
}

\author{
A. Zsom and C. P. Dullemond
}

\author{
Max-Planck-Institut für Astronomie, Königstuhl 17, 69117 Heidelberg, Germany \\ e-mail: [zsom;dullemon] @mpia.de
}

Received 7 April 2008 / Accepted 29 July 2008

\begin{abstract}
Context. There is increasing need for good algorithms for modeling the aggregation and fragmentation of solid particles (dust grains, dust aggregates, boulders) in various astrophysical settings, including protoplanetary disks, planetary- and sub-stellar atmospheres and dense molecular cloud cores. Here we describe a new algorithm that combines advantages of various standard methods into one. Aims. The aim is to develop a method that 1) can solve for aggregation and fragmentation; 2) can easily include the effect and evolution of grain properties such as compactness, composition, etc.; and 3) can be built as a coagulation/fragmentation module into a hydrodynamics simulation where it 3a) allows for non-"thermalized" non-local motions of particles (e.g. movement of particles in turbulent flows with stopping time larger than eddy turn-over time) and $3 \mathrm{~b}$ ) focuses computational effort there where most of the mass is.

Methods. We develop a Monte-Carlo method in which we follow the "life" of a limited number of representative particles. Each of these particles is associated with a certain fraction of the total dust mass and thereby represents a large number of true particles which all are assumed to have the same properties as their representative particle. Under the assumption that the total number of true particles vastly exceeds the number of representative particles, the chance of a representative particle colliding with another representative particle is negligibly small, and we therefore ignore this possibility. This now makes it possible to employ a statistical approach to the evolution of the representative particles, which is the core of our Monte Carlo method.

Results. The method reproduces the known analytic solutions of simplified coagulation kernels, and compares well to numerical results for Brownian motion using other methods. For reasonably well-behaved kernels it produces good results even for moderate number of swarms.
\end{abstract}

Key words. accretion, accretion disks - methods: statistical - planets and satellites: formation - ISM: dust, extinction

\section{Introduction}

Dust particle aggregation is a very common process in various astrophysical settings. In protoplanetary disks the aggregation of dust particles forms the very initial step of planet formation (see e.g. Dominik et al. 2007). It also modifies the optical properties of the disk, and it has influence on the chemistry and free electron abundance in a disk (Sano et al. 2000; Semenov et al. 2004; Ilgner \& Nelson 2006). The appearance and evolution of a protoplanetary disk is therefore critically affected by the dust aggregation process. In sub-stellar and planetary atmospheres the aggregation of dust particles and the coagulation of fluid droplets can affect the structure of cloud layers. It can therefore strongly affect the spectrum of these objects and influence the local conditions within these atmospheres. The process of aggregation/coagulation and the reverse process of fragmentation or cratering are therefore important processes to understand, but at the same time they are extremely complex.

Traditional methods solve the Smoluchowski equation for the particle mass distribution function $f(m)$, where $f(m)$ is defined such that $f(m) \mathrm{d} m$ denotes the number of particles per cubic centimeter with masses in the interval $[m, m+\mathrm{d} m]$. This kind of method has been used in many papers on dust coagulation before (e.g. Nakagawa et al. 1981; Weidenschilling 1984, 1997; Schmitt et al. 1997; Suttner \& Yorke 1999; Tanaka et al. 2005; Dullemond \& Dominik 2005; Nomura \& Nakagawa 2006). Methods of this kind are efficient, but have many known problems. First of all a coarse sampling of the particle mass leads to systematic errors such as the acceleration of growth (Ohtsuki et al. 1990). High resolution is therefore required, which may make certain problems computationally expensive. Moreover, if one wishes to include additional properties of a particle, such as porosity, charge, composition etc., then each of these properties adds another dimension to the problem. If each of these dimensions is sampled properly, this can quickly make the problem prohibitively computationally expensive. Finally, the traditional methods are less well suited for modeling stochastic behavior of particles unless this stochastic behavior can be treated in an averaged way. For instance, in protoplanetary disks if the stopping time of a particle is roughly equal to the turbulent eddy turnover time, then the velocity of a particle with respect to the gas is stochastic: at the same location there can exist two particles with identical properties but which happen to have different velocities because they entered the eddy from different directions (see e.g. the simulations by Johansen et al. 2006).

To circumvent problems of this kind Ormel et al. (2007) have presented a Monte Carlo approach to coagulation. In this approach the particles are treated as computational particles in a volume which is representative of a much larger volume. The simulation follows the life of $N$ particles as they collide and stick or fragment. The collision rates among these particles are computed, and by use of random numbers it is then determined which particle collides with which. The outcome of the collision is then determined depending on the properties of the two 
colliding particles and their relative impact velocity. This method, under ideal conditions, provides the true simulation of the process, except that random numbers are used in combination with collision rates to determine the next collision event. This method has many advantages over the tradiational methods. It is nearly trivial to add any number of particle properties to each particle. There is less worry of systematic errors because it is so close to a true simulation of the system, and it is easy to implement. A disadvantage is that upon coagulation the number of computational particles goes down as the particles coagulate. Ormel et al. solve this problem by enlarging the volume of the simulation and hence add new particles, but this means that the method is not very well suited for modeling coagulation within a spatially resolved setting such as a hydrodynamic simulation or a model of a protoplanetary disk.

It is the purpose of the present paper to present an alternative Monte Carlo method which can quite naturally deal with extremely large numbers of particles, which keeps the number of computational particles constant throughout the simulation and which can be used in spatially resolved models.

\section{The method}

\subsection{Fundamentals of the method}

The fundamental principle underlying the method we present here is to follow the behavior of a limited number of representative particles whose behavior is assumed to be a good representation of all particles. In this approach the number of physical particles $N$ can be arbitrarily large. In fact it should be very much larger than the number of representative particles $n$, so that the chance that one representative particle collides with another representative particle is negligible compared to the collisions between a representative particle and a non-representative particle. In other words, if $N \gg n$, we only need to consider collisions between a representative particle and a non-representative particle. The number of collisions among representative particles is too small to be significant, and the collisions among non-representative particles are not considered because we focus only on the behavior of the representative particles.

Suppose we have a cloud of dust with $N=10^{20}$ physical particles, with a specific size distribution, for instance, MRN (Mathis et al. 1977). Let the total mass of all these particles together be $M_{\text {tot }}$ and the volume be $V$. We randomly pick $n$ particles out of this pool, where $n$ is a number that can be handled by a computer, for instance, $n=1000$. Each representative particle $i$ has its own mass $m_{i}$ and possibly other properties such as porosity $p_{i}$ or charge $c_{i}$ assigned to it. We now follow the life of each of these $n=1000$ particles. To know if representative particle $i=20$ collides with some other object, we need to know the distribution function of all physical particles with which it can collide. However, in the computer we only have information about the $n$ representative particles. We therefore have to make the assumption that the distribution function set up by the $n$ representative particles is representative of that of the $N$ physical particles. We therefore assume that there exist

$n_{k}=\frac{M_{\mathrm{tot}}}{n m_{k} V}$

physical particles per cubic $\mathrm{cm}$ with mass $m_{k}$, porosity $p_{k}$, charge $c_{k}$ etc., and the same for each value of $k$, including $k=i$. In this way, by assumption, we know the distribution of the $N$ physical particles from our limited set of $n$ representative particles. One could say that each representative particle represents a swarm of $M_{\mathrm{tot}} / n m_{i}$ physical particles with identical properties as the representative one. One could also say that the true distribution of $N$ particles is, by assumption, that of the $n$ representative ones. The rate of collisions that representative particle $i$ has with a physical particle with mass $m_{k}$ etc. is then:

$r_{i}(k)=n_{k} \sigma_{i k} \Delta v_{i k}=\frac{M_{\mathrm{tot}}}{n m_{k} V} \sigma_{i k} \Delta v_{i k}$

where $\sigma_{i k}$ is the cross-section for the collision between particles with properties $i$ and $k$, and $\Delta v_{i k}$ is the average relative velocity between these particles. The total rate of collisions that representative particle $i$ has with any particle is then:

$r_{i}=\sum_{k} r_{i}(k)$

and the total rate of collisions of any representative particle is

$r=\sum_{i} r_{i}$

The time-evolution of the system is now done as follows. Let $t_{0}$ be the current time. We now randomly choose a time step $\delta t$ according to:

$\delta t=-\frac{1}{r} \log (\operatorname{ran}(\mathrm{seed}))$

where ran(seed) is a random number uniformly distributed between 0 and 1 . This means that a collision event happens with one of the representative particles at time $t=t_{0}+\delta t$. The chance $P(i)$ that the event happens to representative particle $i$ is:

$P(i)=\frac{r_{i}}{r}$

So we can choose, using again a random number, which representative particle $i$ has undergone the collision event. We now need to determine with what kind of physical particle it has collided. Since the distribution of physical particles mirrors that of the representative ones, we can write that the chance this particle has collided with a physical particle with properties $k$ is:

$P(k \mid i)=\frac{r_{i}(k)}{r_{i}}$.

With another random number we can thus determine which $k$ is involved in the collision. Note that $k$ can be $i$ as well, i.e. the representative particle can collide with a physical particle with the same properties, or in other words: a representative particle can collide with a particle of its own swarm of physical particles.

Now that we know what kind of collision has happened, we need to determine the outcome of the collision. The most fundamental part of our algorithm is the fact that only representative particle $i$ will change its properties in this collision. Physical particle $k$ would in principle also do so (or in fact becomes part of the new representative particle), but since we do not follow the evolution of the physical particles, the collision will only modify the properties of representative particle $i$. By assumption this will then automatically also change the properties of all physical particles associated with representative particle $i$. Statistically, the fact that the particles $k$ are not modified is "corrected for" by the fact that at some point later the representative particle $k$ will have a collision with physical particle $i$, in which case the properties of the $k$ particles will be modified and not those of $i$. This then (at least in a statistical sense) restores the "symmetry" of the interactions between $i$ and $k$. If the collision leads to 
sticking, then the resulting particle will have mass $m=m_{i}+m_{k}$. This means that representative particle $i$ will from now on have mass $m_{i} \leftarrow m_{i}+m_{k}$. Representative particle $k$ is left unaffected as it is not involved in the collision. The interesting thing is now that, because by assumption the representative particle distribution mirrors the real particle distribution, the swarm of physical particles belonging to the modified representative particle $i$ now contains fewer physical particles, because the total dust mass $M=M_{\mathrm{tot}} / n$ of the swarm remains constant.

If a collision results in particle fragmentation, then the outcome of the collision is a distribution function of debris particles. This distribution function can be written as a function $f_{\mathrm{d}}(m)$ of debris particle mass, such that

$$
\int_{0}^{\infty} m f_{\mathrm{d}}(m) \mathrm{d} m=m_{i}+m_{k}
$$

and the function $f_{\mathrm{d}}(m)$ has to be determined by laboratory experiments or detailed computer simulations of individual particle collisions (see Dominik et al. 2007, for a review). The new value of $m_{i}$ for the representative particle is now randomly chosen according to this distribution function by solving the equation

$$
\int_{0}^{\bar{m}} m f_{\mathrm{d}}(m) \mathrm{d} m=\operatorname{ran}(\text { seed })\left(m_{i}+m_{k}\right)
$$

for $\bar{m}$ and assigning $m_{i} \leftarrow \bar{m}$. In other words: we randomly choose a particle mass from the debris mass distribution function, i.e. the choice is weighed by fragment mass, not by fragment particle number. This can be understood by assuming that the true representative particle before the collision is in fact just a monomer inside a larger aggregate. When this aggregate breaks apart into for instance one big and one small fragment it is more likely that this representative monomer resides in the bigger chunk than in the smaller one.

After a fragmenting collision the $m_{i}$ will generally be smaller than before the collision. This means that the number of physical particles belonging to representative particle $i$ increases accordingly. Note that although the collision has perhaps produced millions of debris particles out of two colliding objects, our method only picks one of these debris particles as the new representative particle and forgets all the rest. Clearly if only one such destructive collision happens, the representative particle is not a good representation of this entire cloud of debris products. But if hundreds such collisions happen, and are treated in the way described here, then the statistical nature of Eq. (9) ensures that the debris products are well represented by the representative particles.

The relative velocity $\Delta v$ can be taken to be the average relative velocity in case of random motions, or a systematic relative velocity in case of systematic drift. For instance, for Brownian motion there will be an average relative velocity depending on the masses of both particles, but differential sedimentation in a protoplanetary disk or planetary atmosphere generates a systematic relative velocity. Also, for the Brownian motion or turbulent relative velocity one can, instead of using an average relative velocity, choose randomly from the full distribution of possible relative velocities if this is known. This would allow a consistent treatment of fluctuations of the relative velocities which could under some circumstances become important (see e.g. Kostinski \& Shaw 2005).

\subsection{Computer implementation of the method}

We implemented this method in the following way. For each of the representative particles we store the mass $m_{i}$ and all other properties such as porosity, charge, composition etc. Before the start of the Monte Carlo procedure we compute the full collision rate matrix $r_{i}(k)$, and we compute the $r_{i}$ as well as $r$. For these collision pairs $(i, k)$ we now have to determine the cross section of particles as well as their systematic relative velocity, such as different drift speeds, and the random relative velocity, such as Brownian or turbulent motion. The random motions can be determined with a random number from the relative velocity probability distribution function if that is known. If that is not known in sufficient detail, one can also take it to be the average relative velocity, for which more often analytic formulae exist in the literature.

We determine beforehand at which times $t_{\mathrm{sav}, n}$ we want to write the resulting $m_{i}$ and other parameters to a file. The simulation is now done in a subroutine with a do-while loop. We then determine $\delta t$ using a random number (see Eq. (5)), and check if $t+\delta t<t_{\mathrm{sav}, n}$, where $t_{\mathrm{sav}, n}$ is the next time when the results will have to be stored. If $t+\delta t<t_{\mathrm{sav}, n}$, then a collision event occured before $t_{\mathrm{sav}, n}$. We will handle this event according to a procedure described below, we set $t \leftarrow t+\delta t$ and then return to the point where a new $\delta t$ is randomly determined. If, on the other hand, $t+\delta t \geq t_{\mathrm{sav}, n}$ then we stop the procedure, return to the main program and set $t \leftarrow t_{\mathrm{sav}, n}$. The main program can then write data to file and re-call the subroutine to a time $t_{\mathrm{sav}, n+1}$ or stop the simulation altogether. Note that when the subroutine is called again for a next time interval, it does not need to know the time of the previously randomly determined event which exceeded $t_{\mathrm{sav}, n}$. Of course, one could memorize this time and take that time as the time of the next event in the next time interval, but since the events follow a Poisson distribution, we do not need to know what happened before $t_{\mathrm{sav}, n}$ to randomly determine the new time $t+\delta t$ of the next event.

Now let us turn to what happens if a collision event occurs, i.e. occurs between time $t$ and $t+\delta t$. We then first determine which representative particle $i$ is hit, which is done by generating a random number and choosing from the probability distribution of collision rates, as described in Sect. 2.1. Similarly we determine the non-representative particle with which it collides, or in other words: we determine the index $k$ of the "swarm" in which this non-representative particle resides. Finally, we must determine the impact parameter of the collision, or assume some average impact parameter.

Now we employ a model for the outcome of the collision. This is the collision subroutine of our Monte Carlo method. It is here where the results of laboratory experiments come in, and the translation of such experiments into a coagulation kernel is a major challenge which we do not cover here. The collision model must be a quick formula or subroutine that roughly represents the outcomes of the detailed laboratory collision experiments or detailed numerical collision models. It will give a probability function $f_{\mathrm{d}}$ for the outcoming particle masses and properties. From this distribution function we pick one particle, and from this point on our representative particle $i$ will attain this mass and these properties. The collision partner $k$ will not change, because it is a non-represetative particle from that swarm that was involved in the collision, and we do not follow the life of the non-representative particles. We therefore ignore any changes to that particle.

We now must update $r_{j}(l)$ for all $l$ with fixed $j=i$ and for all $j$ with fixed $l=k$ : we update a row and a column in the $r_{j}(l)$ matrix. Having done this, we must also update $r_{j}$ for all $j$. This would be an $n^{2}$ process, which is slow. But in updating $r_{j}(l)$ we know the difference between the previous and the new value, and we can simply add this difference to $r_{j}$ for each $j$. 
Only for $j=i$ we must recompute the full $r_{j}$ again, because there all elements of that row have been modified. Using this procedure we assure that we limit the computational effort to only the required updates.

\subsection{Acceleration of the algorithm for wide size distributions}

One of the main drawbacks of the basic algorithm described above is that it can be very slow for wide size distributions. Consider a swarm of micron sized dust grains that are motionless and hence do not coagulate among each other. Then a swarm of meter sized boulders moves through the dust swarm at a given speed, sweeping up the dust. Let us assume that also the boulders are not colliding among each other. The only mode of growth is the meter-sized boulders sweeping up the micron sized dust. For the boulder to grow a factor of 2 in mass it will have to sweep up $10^{18}$ micron sized dust particles. Each impact is important for the growth of the boulder, but one needs $10^{18}$ such hits to grow the boulder a factor of 2 in mass. The problem with the basic algorithm described above is that it is forced to explicitly model each one of these $10^{18}$ impacts. This is obviously prohibitively expensive.

The solution to this problem lies in grouping collisions into one. Each impact of a dust grain on a boulder only increases the boulder mass by a minuscule fraction. For the growth of the boulder it would also be fine to lower the chance of an impact by $10^{16}$, but if it happens, then $10^{16}$ particles impact onto the boulder at once. Statistically this should give the same growth curve, and it accelerates the method by a huge factor. However, it introduces a fine-tuning parameter. We must specify the minimum increase of mass for coagulation $\left(\mathrm{d} m_{\max }\right)$. If we set $\mathrm{d} m_{\max }$ to, for instance, $10 \%$, then we may expect that the outcome also has errors of the order of $10 \%$. This error arises because by increasing the mass of the bigger body in steps of $10 \%$, we ignore the fact that the mass at some time should in fact be somewhere in between, which cannot be resolved with this method. This is, however, not a cumulative error. While the mass of the bigger body may sometimes be too low compared to the real one, it equally probably can be too large. On average, by the Poisson nature of the collision events, this averages out. But it is clear that the smaller we take this number, the more accurate it becomes - but also the slower the method becomes. It is therefore always a delicate matter to choose this parameter, but for problems with a large width of the size distribution this acceleration is of vital importance for the usability of the method.

\subsection{Including additional particle properties}

We mentioned briefly the possibility of adding more particle properties to each representative particle. This is very easy to do, and it is one of the main advantages of a Monte Carlo method over methods that directly solve the integral equations for coagulation. One of the main properties of interest to planet formation is porosity or fractal structure of the aggregate. Two aggregates with the same mass can have vastly different behavior upon a collision if they have different compactness. A fluffy aggregate may break apart already at low impact velocities while a compact aggregate may simply bounce. Upon collisions these properties may in fact also change. Ormel et al. (2007) studied the effect of porosity and how it changes over time, and they also used a Monte Carlo approach for it.

If one wishes to include particle properties in a traditional method which solves the integral equations of coagulation (the
Smoluchowski equation), then one increases the dimensionality of the problem by 1 for each property one adds. With only particle mass one has a distribution function $f(m, t)$ while adding two particle properties $p_{1}$ and $p_{2}$ means we get a distribution function $f\left(m, p_{1}, p_{2}, t\right)$, making it a 4-dimensional problem. Methods of this kind must treat the complete phase space spanned by $\left(m, p_{1}, p_{2}, t\right)$. This is of course possible, but computationally it is a very challenging task (see Ossenkopf 1993). In contrast, a Monte Carlo method only sparsely samples phase space, and it samples it only there where a significant portion of the total dust mass is. A Monte Carlo method focuses its computational effort automatically there where the action is. The drawback is that if one is interested in knowing the distribution function there where only very little mass resides, then the method is inaccurate. For instance, in a protoplanetary disk it could very well be that most of the dust mass is locked up in big bodies (larger than $1 \mathrm{~m}$ ) which are not observable, and only a promille of the dust is in small grains, but these small grains determine the infrared appearance of the disk because they have most of the solid surface area and hence most of the opacity. In such a case a Monte Carlo method, by focusing on where most of the mass is, will have a very bad statistics for those dust grains that determine the appearance of the disk. For such goals it is better to use the traditional methods. But if we are interested in following the evolution of the dominant portion of the dust, then Monte Carlo methods naturally focus on the interesting parts of phase space.

\section{Discussion of the method}

\subsection{Conservation of particle number}

There are a few peculiarities of the method described here that may, at first sight, appear inconsistent, but are statistically correct. For instance if we return to our example of a swarm of tiny particles and a swarm of boulders, i.e. $n=2$ with representative particle 1 being a micron sized particle and representative particle 2 being a meter sized particle, then we encounter an apparent paradox. We again assume that collisions only take place between 1 and 2, but not between 1 and 1 or 2 and 2 . The chance that representative particle 1 hits a meter size particle is much smaller than the chance that representative particle 2 hits a micron size particle. What will happen is that representative particle 2 will have very many collision events with small micron size grains, and thereby slowly and gradually grows bigger, while representative particle 1 will only have a collision with big particle after a quite long time and immediately jumps to that big size. While representative particle 2 grows in mass, the number of big physical particles decreases in order to conserve mass. This may seem wrong, because in reality the number of big boulders stays constant, and these boulders simply grow by sweeping up the small dust. The solution to this paradox is that the average time before representative particle 1 hits a big $(k=2)$ particle is of the same order as the time it takes for representative particle 2 to grow to twice its mass by collecting small particles. So, very roughly, by the time the big particle has doubled its mass, and therefore the number of physical particles belonging to $k=2$ has reduced by $50 \%$, the representative particle 1 has turned into a big particle, corresponding, statistically, to the other $50 \%$ of big particles that was missing. If we are a bit more precise, the statistics do not add up precisely in this way if we have only 1 swarm of small and 1 swarm of big bodies. If, however, one has $N$ swarms of small and $N$ swarms of big bodies, and again assume that only the big bodies can sweep up the smaller ones, then if $N \gg 1$ the statistics adds up perfectly: one finds that after 

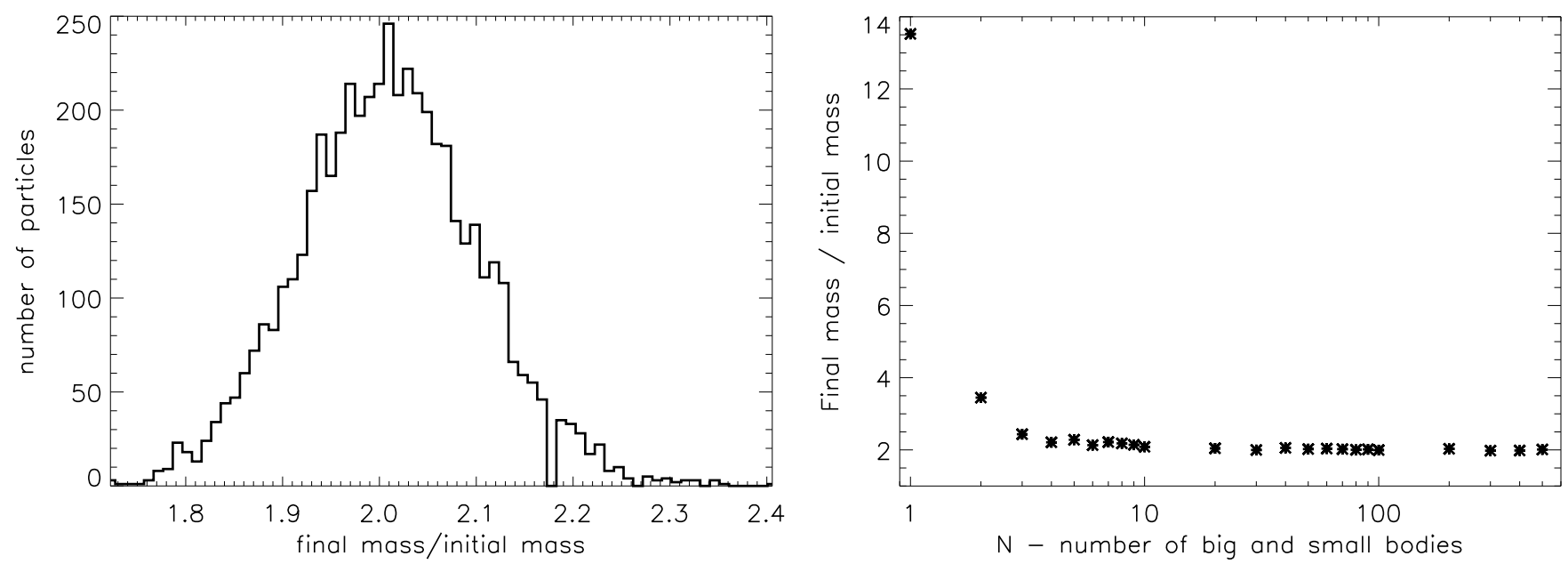

Fig. 1. Results of the test problem with $N$ swarms of small particles and $N$ swarms of big bodies, as discussed in Sect. 3.1. Left: histogram of the final masses of the bodies relative to the initial mass of the big bodies, for $N=500$. Right: average mass relative to the initial mass of the big bodies as a function of $N$.

all the growth has taken place, the average mass of the bodies is twice that of the original big bodies. In Fig. 1 we do precisely this experiment, and the left panel shows that for $N=500$ the mass distribution of the big bodies averages to the right value, albeit with a spread of $10 \%$ FWHM while in reality this spread should be 0 . The right panel shows how the average final mass depends on $N$. For small $N$ the statistics clearly do not add up, but for large $N$ they do and produce the right value (final mass is twice initial mass of the big bodies). So statistically the number of big particles is restored to the correct value, but there is then unfortunately still a large statistical noise on it. The particle number is therefore not exactly conserved in our method, but statistically it is.

\subsection{The number of representative particles}

It is obvious that for high number of representative particles $n$ we will get better results than for low $n$. But there are two issues here. First of all, the higher $n$, the better the representative particles represent the true physical distribution of particles. For problems that result in wide size distributions this is all the more crucial. An inaccurate representation of the true size distribution could lead to systematic errors. But another reason for taking a high $n$ is simply because we want our end-result to have as little as possible noise. If the result is too noisy, then it is useless. Taking $n$ too big, however, makes the code slow because more representative particles have to be followed, and for each of these particles we must check for a larger number of possible collision partners $k$. The problem scales therefore as $n^{2}$. If the expected size distributions are not too wide, one can use an intermediately large number of representative particles, say $\bar{n}$, for the simulation, but redo the simulation $m$ times such that $n=m \bar{n}$, and average the results of all $m$ simulations. This approach was also used by Ormel et al. (2007). This gives the same amount of noise on the end-result, but scales as $\bar{n}^{2} m=n^{2} / m$, which is $m$ times faster than the $n^{2}$ scaling. This works, however, only if the coagulation/fragmentation kernel is not too sensitive to the exact distribution of collision partners.

Interestingly, if the kernel is very insensitive to the exact distribution of collision partners, then, in principle, one could run the model with only a single representative particle $n=1$, because the collision partner of representative particle $i$ could be equal to $k=i$. Of course, a single representative particle means that we assume that all physical particles have the same size, or in other words: that we have an infinitely narrow size distribution.

To decide about the sufficient number of representative particles, one has to compare the results of the MC code with the analytical solutions of the three test kernels (see Sect. 4). In a given time of the simulation the mean mass and the shape of the distribution function for all three test kernels must be followed accurately. It is especially important to reproduce the linear and product kernels accurately as the realistic kernels of dust particles are similar to these.

Of course the progression from "not sufficient" and "sufficient" number of representative particles is smooth and in general the more representative particles we use, the more accurate the produced result will be. The sufficient number of representative particles $(\bar{n})$ as given in Sect. 4 are only suggestions, the error of the distribution functions were not quantified.

\subsection{Limitations of the method}

One of the fundamental limitations of the method described here is that we assume $N \gg n$. We can model the growth of particles by coagulation in a protoplanetary disk or in a cloud in a planetary atmosphere, but we can not follow the growth to the point where individual large bodies start to dominate their surroundings. For instance, if we wish to follow the growth of dust in a protoplanetary disk all the way to small planets, then the method breaks down, because $N$ is then no longer much bigger than $n$, and interactions among representative particles become likely. Also, for the same reason, run-away growth problems such as electrostatic gelation (Mokler 2007) cannot be modeled with this method.

Another limitation is encountered when modeling problems with strong growth and fragmentation happening at the same time. This leads to very wide size distributions, and the typical interval between events is then dominated by the smallest particles, whereas we may be interested primarily in the growth of the biggest particles. In such a situation a semi-steady-state can be reached in which particles coagulate and fragment thousands of times over the life time of a disk. The Monte Carlo method has to follow each of these thousands of cycles of growth and destruction, which makes the problem very "stiff". Methods using the integral form of the equations, i.e. the Smoluchowski 
equation, can be programmed using implicit integration in time so that time steps can be taken which are much larger than the typical time scale of one growth-fragmentation cycle without loss of accuracy (Brauer et al. 2008). This is not possible with a Monte Carlo method.

\section{Standard tests and results}

In this section we test our coagulation model with kernels that have analytical solutions. Furthermore we show the first results of applying this model to protoplanetary disks introducing Brownian motion and turbulence induced relative velocities as well as a new property of dust particles namely the porosity (or enlargement factor, see Ormel et al 2007), and a simple fragmentation model.

To follow dust coagulation and fragmentation, one has to follow the time evolution of the particle distribution function at a given location in the disk $(f(\boldsymbol{y}, t))$, where $\boldsymbol{y}$ contains the modeled properties of the dust grains, in our case these will be the mass $(m)$ and the enlargement factor $(\Psi), f(m, \Psi, t)$.

In most of the coagulation models so far the only used dustproperty was the particle mass. Then one can use the so called Smoluchowski equation (Smoluchowski 1916) to describe the time-evolution of $f(m)$ :

$$
\begin{aligned}
\frac{\partial f(m)}{\partial t}=-f( & m) \int \mathrm{d} m^{\prime} K\left(m, m^{\prime}\right) f\left(m^{\prime}\right) \\
& +\frac{1}{2} \int \mathrm{d} m^{\prime} K\left(m^{\prime}, m-m^{\prime}\right) f\left(m^{\prime}\right) f\left(m-m^{\prime}\right) .
\end{aligned}
$$

The first term on the right hand side represents the loss of dust in the mass bin $m$ by coagulation of a particle of mass $m$ with a particle of mass $m^{\prime}$. The second term represents the gain of dust matter in the mass bin $m$ by coagulation of two grains of mass $m^{\prime}$ and $m-m^{\prime} . K$ is the coagulation kernel, it can be written as

$K\left(m_{1}, m_{2}\right)=\sigma_{\mathrm{c}}\left(m_{1}, m_{2}\right) \times \Delta v\left(m_{1}, m_{2}\right)$,

the product of the the cross-section of two particles and their relative velocity. We consider all the three kernels for which there exist analytical solutions: the constant $\operatorname{kernel}\left(K_{i, j}=1\right)$, the linear $\operatorname{kernel}\left(K_{i, j}=m_{i}+m_{j}\right)$ and the product $\operatorname{kernel}\left(K_{i, j}=m_{i} \times m_{j}\right)$. The analytical solutions are described e.g. in Ohtsuki et al. (1990) and Wetherill (1990).

We test our method against these three kernels, leaving the enlargement factor unchanged, always unity. Further important properties of the dust particles, such as material density and volume density, are also always unity. The (dimensionless) time evolution of the swarms is followed and at given times the particles are binned by mass so that we can produce $f(m)$. In Figs. 2 and 3 the $y$ axis shows $f(m) \times m^{2}$, the mass density per bin. The analytical solutions, taken from Ohtsuki et al. (1990) and Wetherill (1990), are overplotted with dashed line. The number of particles were chosen to be $\bar{n}=200, m=5$, so altogether 1000 representative particles were used in the model except for the product kernel where more representative particles were used to achieve better results.

In the case of the constant kernel (Fig. 2), we started our simulation with MRN size distribution $\left(n(a) \propto a^{-3.5}\right)$, the results were saved at $t=0,10^{0}, 10^{1}, 10^{2}, 10^{3}, 10^{4}, 10^{5}$. It is interesting to note that this kernel is not sensitive to the initial size distribution. As the system evolves, it forgets the initial conditions. Another interesting property of this kernel that our model can reproduce the analytical solution even with very limited number of representative particles (even for $\bar{n}=5$ !) but of course

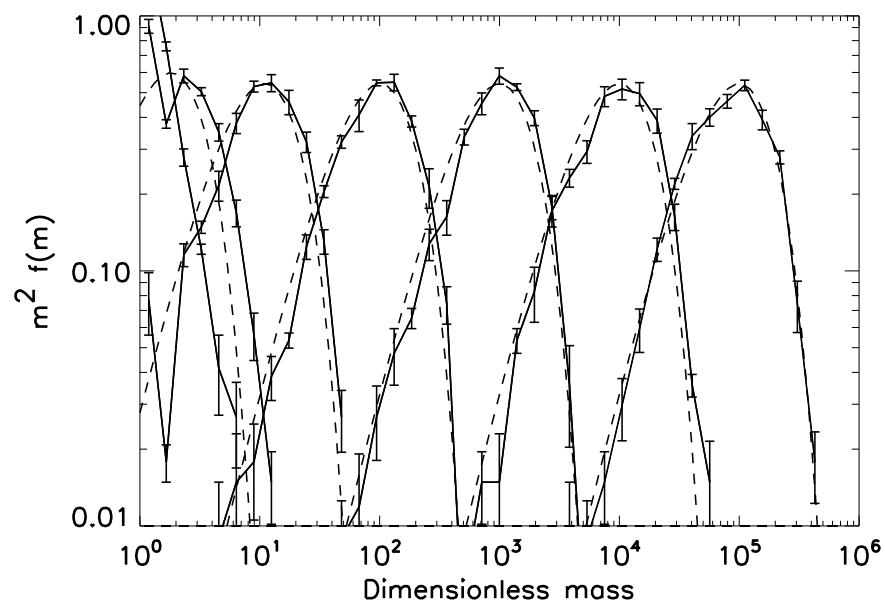

Fig. 2. Test against the constant kernel $\left(K_{i, j}=1\right)$. The particles were binned and the distribution function was produced at dimensionless times $t=0,10^{0}, 10^{1}, 10^{2}, 10^{3}, 10^{4}, 10^{5}$. The dashed lines show the analytical solution. This run was produced by simulating 200 representative particles five times and producing the average of these. In this case $\mathrm{d} m_{\max }$ is 0.1 .

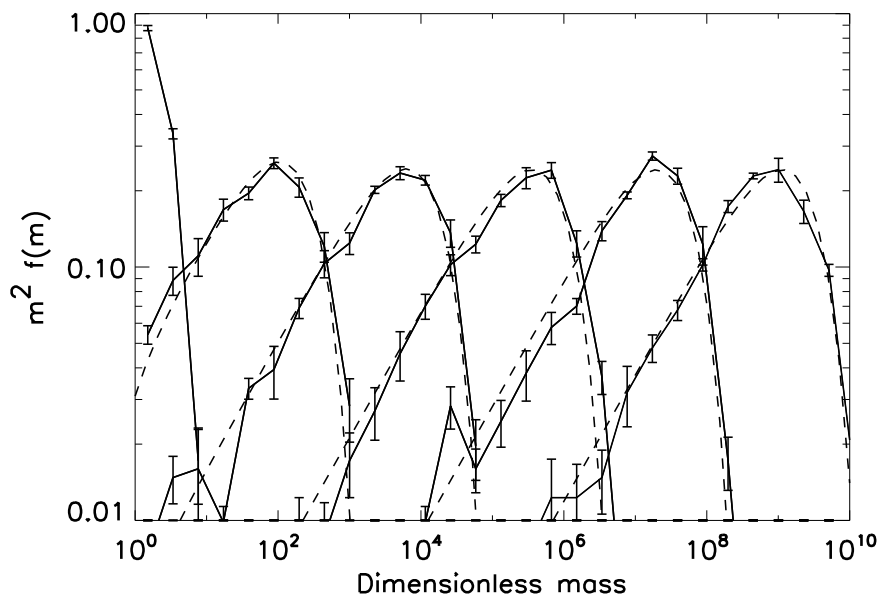

Fig. 3. Test against the linear kernel $\left(K_{i, j}=m_{i}+m_{j}\right)$. The particles were binned and the distribution function was produced at dimensionless times $t=0,4,8,12,16,20$. The dashed lines show the analytical solution. This run was also produced by simulating 200 representative particles five times and producing the average of these. In this case $\mathrm{d} m_{\max }$ is 0.1 .

with higher noise. It is possible to use only one representative particle, which means that the representative particle collides with particles from its own swarm which basically results in pure CCA growth (Cluster-Cluster Aggregation). Interestingly, the mean mass of the distribution function is followed correctly but the shape of the function changes, additional spikes appear on it.

The linear kernel is known to be more problematic because the mean mass of the particles grows exponentially with time. Our model, however reproduces this kernel very well, too, as it can be seen in Fig. 3. The results were saved at $t=0,4,8,12,16,20$. We note that using low number of representative particles with this kernel also works relatively well, the minimum number of swarms needed to reproduce the exponential time evolution of the mean mass is $\bar{n} \approx 100$. This is larger than for the constant kernel. It shows that for the linear kernel collisions between particles of unequal mass are contributing significantly to the growth, whereas for the constant 


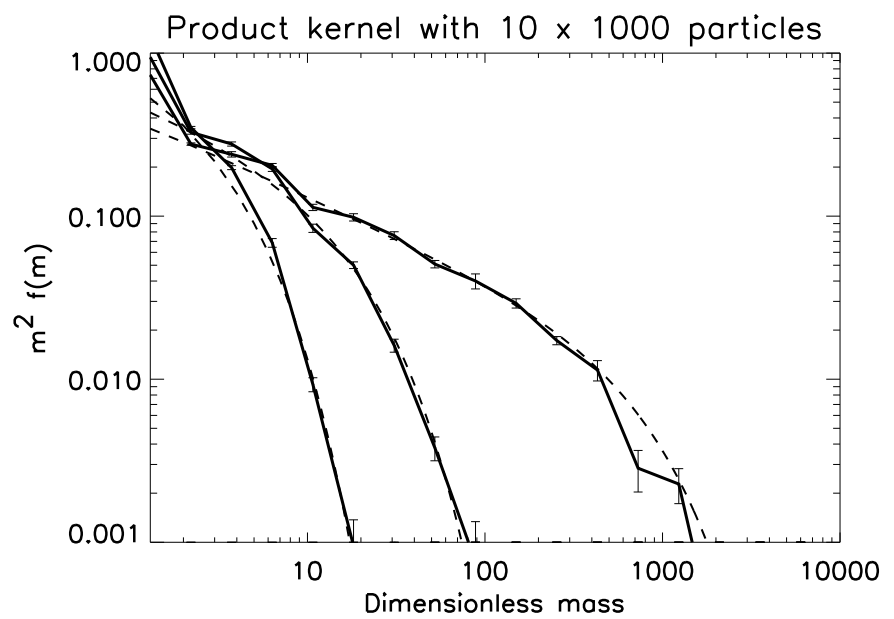

Fig. 4. Test against the product kernel $\left(K_{i, j}=m_{i} \times m_{j}\right)$. The particles were binned and the distribution function was produced at dimensionless times $t=0.4,0.7,0.95$. The dashed lines show the analytical solution. This run was produced by simulating 1000 representative particles ten times and producing the average of these. In this case $\mathrm{d} m_{\max }$ is set to be 0.05 .

kernel the growth is dominated by collisions between roughly equal size particles. Using $\bar{n} \ll 100$ results in distorted distribution function: neither the mean mass nor the actual shape of the distribution function is correct.

The product kernel is the hardest to reproduce. The peculiarity of this kernel is the following: using dimensionless units, a "run-away" particle is produced around $t=1$, which collects all the other particles present in the simulation (Wetherill 1990). The difficulty arises in our Monte Carlo code when the mass of the representative "run-away" particle reaches the mass of its swarm. In other words, the number of physical particles belonging to the representative "run-away" particle is close to unity. In this case the original assumption of our method (we only need to consider collisions between a representative particle and a physical particle) is not valid anymore. However, as Fig. 4 shows, we can relatively well reproduce this kernel before $t=1$. In the case of this kernel, we need approximately $\bar{n} \approx 500$ representative particles to correctly reproduce it.

The required CPU time for these test cases is very low, some seconds only.

We conclude that our Monte Carlo method reproduces the constant and linear test kernels without any problem even with low number of representative particles. On the other hand the method has difficulties with the product kernel, but before the formation of the "run-away" particle, we can reproduce the kernel. The relatively low number of representative particles needed to sufficiently reproduce the test kernels is very important for future applications where whole disk simulations will be done and there will likely be regions containing low numbers of particles.

\section{Applications to protoplanetary disks}

We use the Monte Carlo code to follow the coagulation and fragmentation of dust particles in the midplane of a protoplanetary disk at 1 AU from the central star. Our disk model is identical with the one used by Brauer et al. (2007). We proceed step by step. First relative velocities induced by Brownian motion and turbulence without the effects of porosity are included (Sect. 5.1).

The next step is to include a fragmentation model (Sect. 5.2).

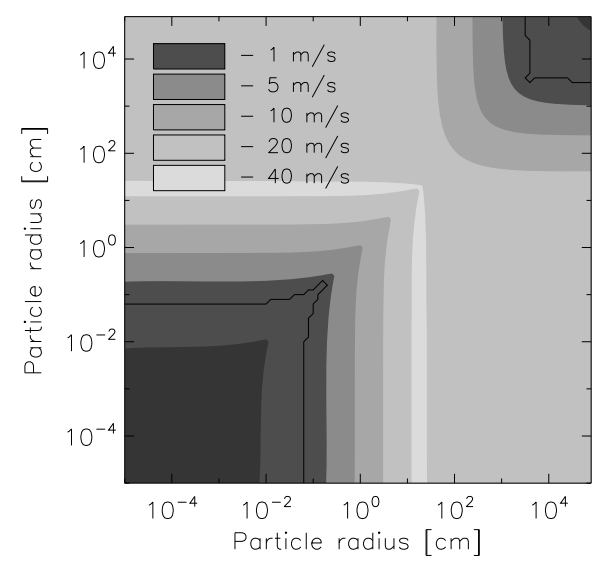

Fig. 5. The relative velocity caused by Brownian motion and turbulence for different sized particles. The black line shows the fragmentation barrier. Collision events situated between these two lines result in fragmentation if porosity is not included. Physical parameters of the disk: the distance from the central star is $1 \mathrm{AU}$, temperature is $200 \mathrm{~K}$, the density of the gas is $8.73 \times 10^{12}$ particle $/ \mathrm{cm}^{3}$, and the turbulent parameter, $\alpha=10^{-3}$. Parameters of the dust: monomer radius is $a_{0}=0.4 \mu \mathrm{m}$, material density is $\rho=1.6 \mathrm{~g} / \mathrm{cm}^{3}$.

In the final step porosity is included (Sect. 5.3). We use the porosity model described in Ormel et al. (2007). At this point we compare and check again our code with Ormel et al. (2007) using their input parameters but not including the rain out of particles.

\subsection{Relative velocities}

We include two processes in calculating the relative velocities: Brownian motion and turbulence.

Brownian motion strongly depends on the mass of the two colliding particles. The smaller their masses are, the more they can be influenced by the random collisions with the gas molecules/atoms. One can calculate an average velocity given by

$$
\Delta v_{\mathrm{B}}\left(m_{1}, m_{2}\right)=\sqrt{\frac{8 k T\left(m_{1}+m_{2}\right)}{\pi m_{1} m_{2}}}
$$

For micron sized particles, relative velocity can be in the order of magnitude of $1 \mathrm{~cm} / \mathrm{s}^{-1}$, but for $\mathrm{cm}$ sized particles this value drops to $10^{-7} \mathrm{~cm} / \mathrm{s}^{-1}$. If growth is only governed by Brownian motion, it leads to very slow coagulation, a narrow size distribution and fluffy dust particles, so called cluster-cluster aggregates (CCA).

The gas in the circumstellar disk is turbulent, thus the dust particles experience acceleration from eddies with different sizes and turnover times. This process is very complex, but Ormel \& Cuzzi (2007) provided limiting closed-form expressions for average relative turbulent velocities between two dust particles. Their results are also valid for particles with high Stokes numbers. They distinguished three regimes: a) the stopping times of both dust particles are smaller than the smallest eddy-turnover time $\left(t_{1}, t_{2}<t_{\eta}\right.$, tightly coupled particles); b) the stopping time is between the smallest and largest turnover time $\left(t_{\eta} \leq t_{1} \leq t_{\mathrm{L}}\right.$, intermediate regime); c) the stopping time is bigger than the largest turnover time $\left(t_{1}>t_{\mathrm{L}}\right.$, heavy particles). For details see Ormel \& Cuzzi (2007). We used $\alpha=10^{-3}$ for the turbulence parameter.

To illustrate the relative velocity of dust particles without the effects of porosity, we provide Fig. 5. This contour plot includes Brownian motion and turbulent relative velocities. The Brownian motion is negligible for particles bigger than $10^{-2} \mathrm{~cm}$. 


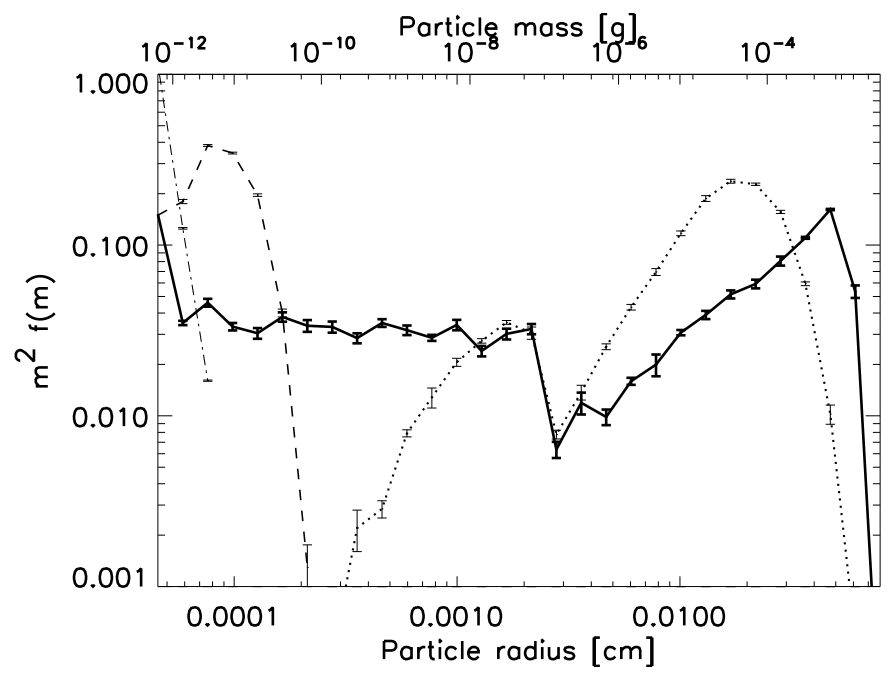

Fig. 6. The evolution of dust particles including the effects of Brownian motion and turbulence. Porosity is not included in this model. The particle distribution is saved after $t=3 \times 10^{\circ}$ years - dash-dot line, $3 \times 10^{1}$ years - dashed line, $3 \times 10^{2}$ years - dotted line, and $3 \times 10^{3}$ years - continuous line.

\subsection{Fragmentation model}

The collision energy of the particles is

$E=\frac{1}{2} \frac{m_{1} m_{2}}{m_{1}+m_{2}} \Delta v^{2}=\frac{1}{2} \mu \Delta v^{2}$,

where $\mu$ is the reduced mass. We need to define some quantities of the dust particles. $E_{\text {roll }}$ is the rolling energy of two monomers. For monomers of the same size it is given by (Dominik \& Tielens 1997; Blum \& Wurm 2000)

$E_{\text {roll }}=\frac{1}{2} \pi a_{0} F_{\text {roll }}$,

where $a_{0}$ is the monomer radius, $F_{\text {roll }}$ is the rolling force measured by Heim et al. (1999). Its value is $F_{\text {roll }}=(8.5 \pm 1.6) \times$ $10^{-5}$ dyn for $\mathrm{SiO}_{2}$ spheres.

The fragmentation energy is then defined as follows:

$E_{\text {frag }}=N_{\mathrm{c}} \times E_{\text {break }} \simeq 3 N \times E_{\text {roll }}$,

where $N_{\mathrm{c}}$ is the total number of contact surfaces between monomers (for simplicity it is taken to be $3 N$, where $N$ is the number of monomers in the particle), $E_{\text {break }}$ is the energy needed to break the bond between two monomers (its order of magnitude is similar to $E_{\text {roll }}$ for these parameters).

If the collision energy of two particles is higher than the corresponding fragmentation energy, then the aggregate is destroyed and monomers are produced. Note that although assuming a complete destruction of the collided dust particles, we are interested in the critical energy where the first fragmentation event happens. This is the reason why the fragmentation energy is assumed to be lower than the energy needed for catastrophic fragmentation. It is a simplification of the model to assume that the debris particles will be monomers. This is a very simplified fragmentation model used previously by Dullemond \& Dominik (2005). A more realistic model would be the one used by Brauer et al. (2007).

We show the fragmentation barrier in Fig. 5 with black lines. If collision happens in the regime between these two lines, that results in fragmentation.

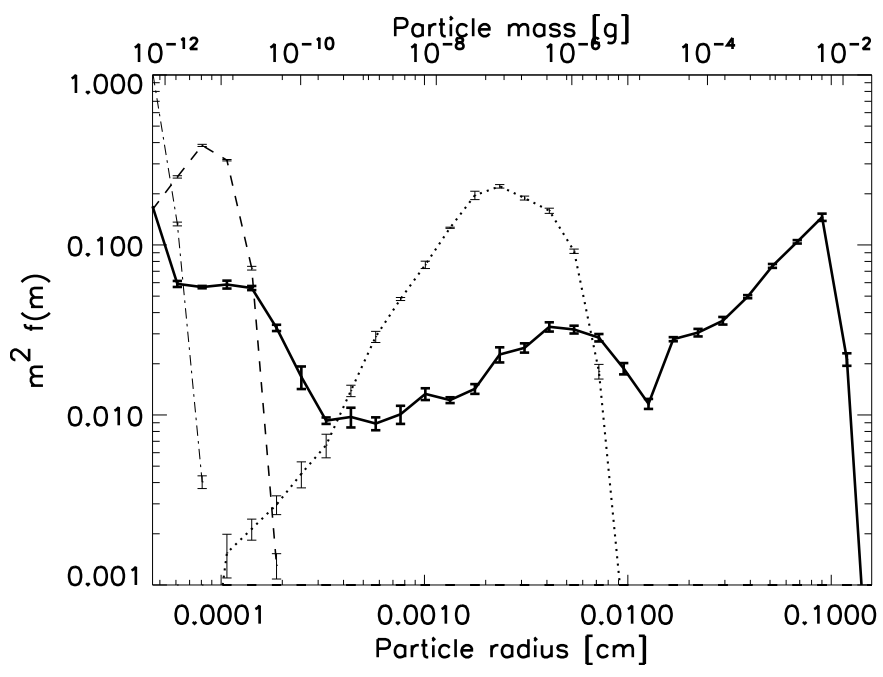

Fig. 7. The evolution of dust particles including the effects of Brownian motion and turbulence. Porosity is included in this model! The $x$ axis shows the compact radius. The particle distribution is saved after $t=$ $3 \times 10^{0}$ years - dash-dot line, $3 \times 10^{1}$ years - dashed line, $3 \times 10^{2}$ years - dotted line, and $3 \times 10^{3}$ years - continuous line. Note that the scaling of the $x$ axis is different from Fig. 6.

\subsubsection{Results}

A simulation was made including these effects in a specified location of the disk. We choose the location to be 1 AU distance from the central solar type star. Using the disk model of Brauer et al. (2007), the temperature at this distance is approximately $200 \mathrm{~K}$, the density of the gas is $8.73 \times 10^{12} \mathrm{~cm}^{-3}$, the gas-todust ratio is 100 and we choose the turbulent parameter to be $\alpha=10^{-3}$, the Reynolds number is $\operatorname{Re}=10^{8}$ (based on Ormel $\&$ Cuzzi 2007). The dust monomers have the following properties: the monomer radius is $a_{0}=0.4 \mu \mathrm{m}$, material density is $\rho=1.6 \mathrm{~g} / \mathrm{cm}^{3}$. With the used parameters the fragmentation velocity is $\Delta v_{\text {frag }} \approx 8 \mathrm{~m} / \mathrm{s}$, though it is somewhat larger for equal sized agglomerates. It is important to note that this value is very sensitive to the monomer radius $\left(a_{0}\right)$ and material density $(\rho)$, because smaller/lighter monomers mean more contact surfaces (higher $N$ for the same mass) and therefore higher fragmentation energy.

Using these input parameters we simulated the evolution of the dust particles for $3 \times 10^{3}$ years so that we reach an equilibrium between coagulation and fragmentation. Figure 6 shows the resulting normalized size distributions in times after $t=3 \times 10^{0}$, $3 \times 10^{1}, 3 \times 10^{2}$ and $3 \times 10^{3}$ years. We used $\bar{n}=100$ particles averaging over $m=100$ times $\left(10^{4}\right.$ particles altogether $)$. The required CPU time to perform this simulation is $1.5 \mathrm{~h}$ approximately. $\mathrm{d} m_{\max }$ is set to be 0.001 from now on in every simulation. We would like to note that giving $\mathrm{d} m_{\max }$ (Sect. 2.3) a higher value would decrease the CPU time.

One can see that coagulation happens due to Brownian motion in the beginning of the simulation (until $3 \times 10^{1}$ years) but after that turbulence takes over and the first fragmentation event happens after roughly $10^{3}$ years. After this event the "recycled" monomers start to grow again, but as we see in Fig. 5, particles can not reach bigger sizes than $0.07 \mathrm{~cm}$.

We would like to draw attention to the sudden decrease of particles around $0.002 \mathrm{~cm}$ in Fig. 6. This is the result of the turbulent relative velocity model used here (discussed in Sect. 5.1). At this point the particles leave the "tightly coupled particles" regime and enter the "intermediate" regime. But the transition 


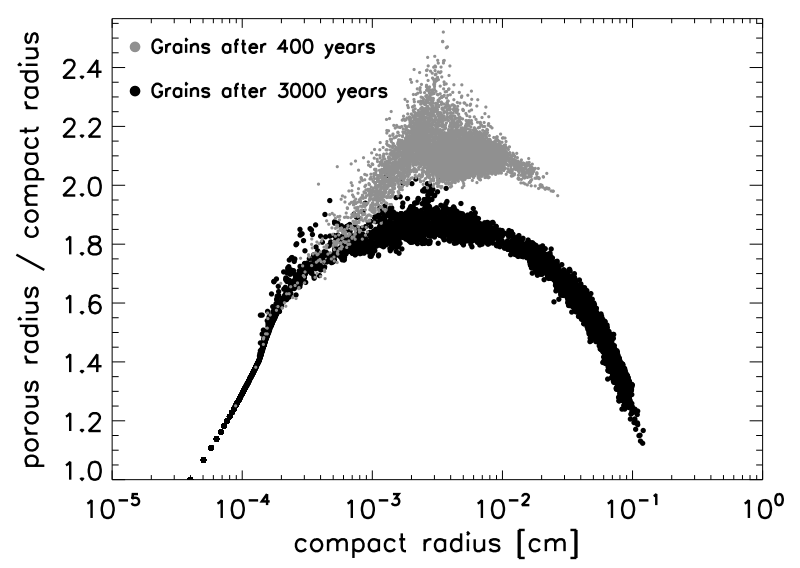

Fig. 8. This figure shows the radial enlargement of the dust aggregates after 400 and 3000 years. The $x$ axis is the compact radii of the particles, the $y$ axis is the ratio between the compact and the porous radius, this quantity is basically equal to $\Psi^{\frac{1}{3}}$.

in relative velocity between these regimes is not smooth, there is a jump in relative velocity from $\sim 20 \mathrm{~cm} / \mathrm{s}$ to $\sim 60 \mathrm{~cm} / \mathrm{s}$. As a result, particles coagulate suddenly faster and leave this part of the size distribution rapidly. Similar "valleys" can be seen in the following figures with porosity, but the feature is less distinct as the stopping times can be different for particles with same mass.

\subsection{Porosity}

To be able to quantitatively discuss the effect of porosity, we have to define the enlargement parameter following the discussion of Ormel et al. (2007). If $V$ is the extended volume of the grain and $V^{*}$ is the compact volume, than one can define the enlargement parameter $(\Psi)$ as

$\Psi=\frac{V}{V^{*}}$.

Compact volume is the volume occupied by the monomers not taking into account the free space between the monomer spheres. One can think of it as melting all the monomers into a single sphere, the volume of this sphere is the compact volume. We use compact radius later on, which is the radius of this sphere. In the previous section the mass/volume ratio was constant for the particles. Therefore we could automatically calculate the mass of the particle if the radius was known or vice-versa. But from now on a particle with given mass $m$ can have a wide range of effective radii depending on its enlargement parameter.

It is essential to know how the enlargement parameter changes upon collisions. We have to refine our fragmentation model and introduce two more regimes regarding to collision energy. We use the model of Ormel et al. (2007) and we only summarize their model here.

The first regime is the low collision energy regime, where the collision energy is smaller than the restructuring energy $\left(E<E_{\text {restr }}\right.$, where $\left.E_{\text {restr }}=5 E_{\text {roll }}\right)$, meaning that the particles stick where they meet, the internal structure of the grain does not change.

The recipe for the resulting enlargement factor after the collision of two particles assuming that $m_{1}>m_{2}$ then is

$\Psi=\langle\Psi\rangle_{m}\left(1+\frac{m_{2} \Psi_{2}}{m_{1} \Psi_{1}}\right)^{\frac{3}{2} \delta_{\mathrm{CCA}}-1}+\Psi_{\mathrm{add}}$, where $\langle\Psi\rangle_{m}$ is the mass averaged enlargement factor of the colliding particles:

$\langle\Psi\rangle_{m}=\frac{m_{1} \Psi_{1}+m_{2} \Psi_{2}}{m_{1}+m_{2}}$.

Furthermore $\delta_{\mathrm{CCA}}$ is the CCA-characteristic exponent calculated by detailed numerical studies such as Paszun \& Dominik (2006) $\left(\delta_{\mathrm{CCA}}=0.95\right) . \Psi_{\text {add }}$ is a necessary additional factor for the enlargement factor (for details see Ormel et al. 2007):

$\Psi_{\text {add }}=\frac{m_{2}}{m_{1}} \Psi_{1} \exp \left[\frac{-\mu}{10 m_{0}}\right]$,

where $m_{0}$ is the monomer mass.

The second regime is the regime of compaction. The internal structure of the monomers inside the particle changes, this causes a decreasing porous volume. If the collision energy $E_{\text {restr }} \leq E \leq E_{\text {frag }}$, we talk about compaction. In this case the porosity after the collision becomes

$\Psi=\left(1-f_{\mathrm{C}}\right)\left(\langle\Psi\rangle_{m}-1\right)+1$,

where $f_{\mathrm{C}}=E /\left(N E_{\mathrm{roll}}\right)=-\Delta V / V$ is the relative compaction. One can see that $f_{\mathrm{C}}$ has to be smaller than unity otherwise $\Psi$ in Eq. (20) becomes less than unity. But it can theoretically happen that $E>N E_{\text {roll }}$. In this case, as long as the total collision energy remains below the fragmentation threshold, we assume that after compaction this excess energy goes back into the kinetic energy of the two colliding aggregates. The two aggregates therefore compactify and bounce, without exchanging mass or being destroyed. Bouncing is therefore included in this model, albeit in a crude way.

The third regime is fragmentation as it was discussed in the previous section ( $E>E_{\text {frag }}$ ). We use the same fragmentation model as before so the result of a fragmenting collision are monomers.

\subsubsection{Results}

We performed a simulation with exactly the same initial conditions as in the last section but we included the porosity as an additional dust property in the model. The result can be seen in Fig. 7 (the required CPU time here is also $1.5 \mathrm{~h}$ ). One can immediately see that including porosity increases the maximum particle mass by two orders of magnitude (five times larger particles in radius). This was already expected based on the work of Ormel et al. (2007), although due to rain out of bigger particles, they did not simulate particles bigger than $0.1 \mathrm{~cm}$.

We provide Fig. 8 to give an impression how the porosity of the agglomerates change during the simulation. The $x$ axis is the compact radius of the particles, the $y$ axis is the ratio between the compact and the porous radii. This quantity is basically equal to $\Psi^{\frac{1}{3}}$. Fractal growth is important for small particles creating fluffy agglomerates (until $10^{-3}-10^{-2} \mathrm{~cm}$ approximately), after this point the relative velocities become high enough so compactness becomes important. Before the particles reach a fully compacted stage they fragment, become monomers and a new cycle of growth starts. It is important to note that the porosity of the aggregates before the first fragmentation event is usually higher than the porosity values after equilibrium is reached. This can be seen in Fig. 8 (grains after 400 years and 3000 years). The reason is that before the first fragmentation event, particles involved in collisions are typically equal sized so these particles produce fluffy structures. However, when the distribution function relaxes in equilibrium, there are collisions between smaller 
and bigger aggregates as well which results in somewhat compacted aggregates.

\subsubsection{Model comparison with Ormel et al. (2007)}

We compare our Monte Carlo code with the one developed by Ormel et al. (2007). They use the Minimum Mass Solar Nebula disk model (MMSN) and somewhat different dustparameters which we changed accordingly (distance from the central star $=1 \mathrm{AU}$, temperature $=280 \mathrm{~K}$, density of the gas is $8.5 \times 10^{-10} \mathrm{~g} / \mathrm{cm}^{3}$, gas to dust ratio $=240, \alpha=10^{-4}$; monomer radius $=0.1 \mu \mathrm{m}$, monomer density $=3 \mathrm{~g} / \mathrm{cm}^{3}$, surface energy density of the monomers $=25 \mathrm{erg} / \mathrm{cm}^{-2}$ ).

They follow particle coagulation at one pressure scale height above the midplane of the disk. Because of this if the particles reach a critical stopping time $\left(\tau_{\text {rain }}=\alpha / \Omega\right.$, where $\Omega$ is the Kepler frequency), the particles rain out meaning that these particles leave the volume of the simulation, the distribution function of the dust particles is collapsing as it can be seen in their figures (Figs. 10 and 11 in Ormel et al. 2007).

We do not include this effect in our model but we stop the simulation at the first rain out event and compare our distribution functions until this point. We use $10^{4}$ representative particles $(100 \times 100)$ during the simulation.

This can be seen at Fig. 9. The reader is advised to examine this figure together with Fig. 10c from Ormel et al. (2007) because this is the figure we reproduced here. Furthermore we would like to point out that the scale of the $y$ axis is different in the two figures. Our figure shows two orders of magnitude from the normalized distribution functions whereas their figure covers more than 10 orders of magnitude from the real distribution function.

Keeping these in mind, one can compare the results of the two Monte Carlo codes.

The continuous lines at Fig. 9 in this paper show the distribution functions at $t=10$ years (thin line), 100 years (thicker line), 1000 years (thickest line). The dotted line shows the distribution function at the time of the first compaction event $(t=$ 1510 years), the dashed line shows the distribution function at the first rain out event ( $t=2900$ years). The same notation is used by Ormel et al. (2007) at Fig. 10c.

We compared the position of the peaks of the distribution functions and the approximate shape of the curves. We can conclude that our code reproduces the results of Ormel et al. (2007) very well.

The required CPU time to perform this simulation is only 10 min. One might ask why the CPU time is almost ten times smaller now? Why do the previous simulations, which used the same number of representative particles $\left(10^{4}\right)$ and simulated approximately the same time interval (3000 years), take so long? The required CPU time does not scale linearly with the used number of particles. It scales linearly with the number of collisions simulated. The difference between this run and the previous two simulations is fragmentation. In the simulations of Ormel et al. (2007) no fragmentation is happening because the growth timescales are longer. Using our initial parameters, the first fragmentation event happens around 1000 years, the number of small particles are never completely depleted after this time. As the small particles thereafter are always present, the number of collisions will be much higher than before.

Also note that the porosities of these particles would be smaller if the model of Ormel et al. (2007) included fragmentation (for the reason see Sect. 5.3.1).

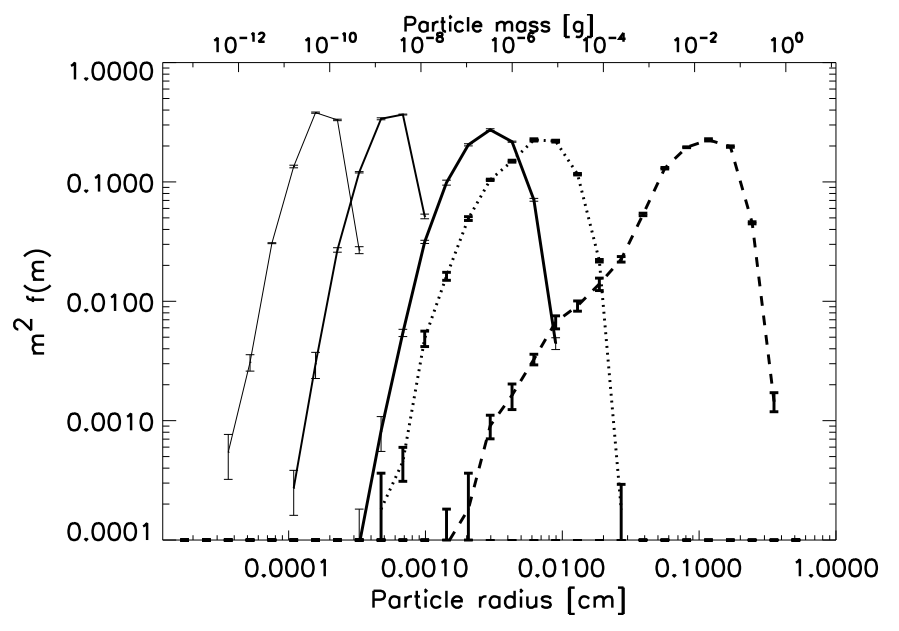

Fig. 9. Distribution functions obtained by using Ormel et al. (2007) input parameters. The continuous lines show the distribution functions at $t=10$ years (thin line), 100 years (thicker line), 1000 years (thickest line). The dotted line shows the distribution function at the time of the first compaction event ( $t=1510$ years), the dashed line shows the distribution function at the first rain out event ( $t=2900$ years).

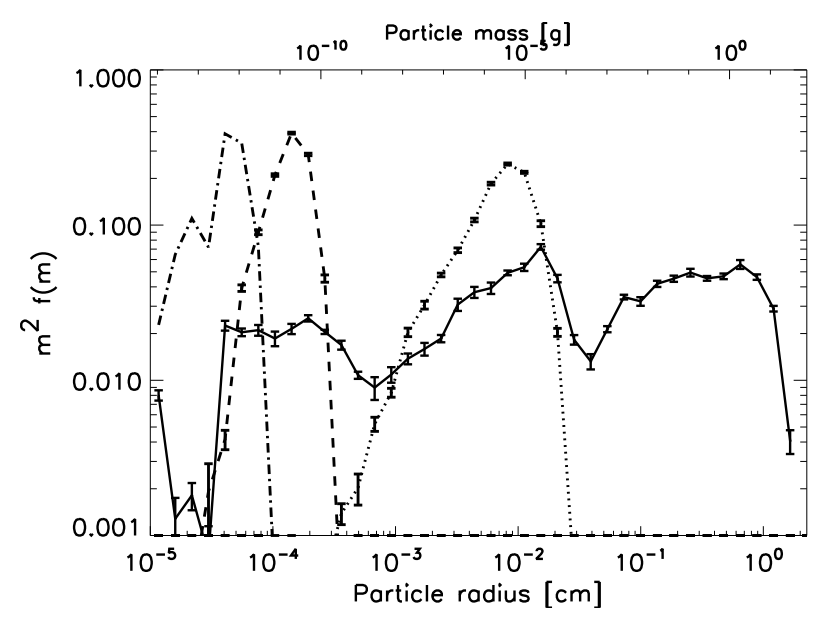

Fig. 10. The evolution of dust particles including the effects of Brownian motion and turbulence, porosity and using two different monomer sizes $\left(a_{1}=0.1 \mu \mathrm{m}\right.$ and $\left.a_{2}=0.4 \mu \mathrm{m}\right)$. The particle distribution is saved after $t=3 \times 10^{0}$ years - dash-dot line, $3 \times 10^{1}$ years - dashed line, $3 \times 10^{2}$ years - dotted line, and $3 \times 10^{3}$ years - continuous line.

\subsection{Monomer size distribution}

An interesting question which can easily be answered with our method is: how the mixture of different sized monomers change the maximum agglomerate size which can be reached? As we can see from Eqs. (14) and (15), the rolling energy is lower for smaller monomers and of course the number of monomers in an agglomerate is much higher if the same agglomerate is built up of lighter monomers. This would mean higher fragmentation energy and one would expect that the particles would be harder to fragment resulting in bigger grains.

We performed a simplified simulation to be able to answer this question. Only two different monomer sizes are considered here, $a_{1}=0.1 \mu \mathrm{m}$ and $a_{2}=0.4 \mu \mathrm{m}$ assuming that half of the mass (or representative particles) belongs to the small monomers, the other half belongs to the big monomers. 
One problem arises here with the rolling energy. The rolling energy changes with monomer size, and as our method cannot follow exactly the number of contacts in an aggregate and what kind of monomers are connected, we are forced to use an averaged rolling energy. One has to carefully consider what the average rolling energy should be. In our case, the big monomer is 64 times heavier than the small monomer. Let's assume that $50 \%$ of the mass of an aggregate is built up from small monomers; on the other hand, if we compare the number of different monomers, the small monomers will be 64 times more numerous than the big ones. This means that the contribution of small monomers in the average rolling energy $\left(\bar{E}_{\text {roll }}\right)$ should be higher. This can be achieved by using the following weighting:

$\bar{E}_{\mathrm{roll}}=\frac{a_{1}}{a_{1}+a_{2}} E_{\mathrm{roll}_{2}}+\frac{a_{2}}{a_{1}+a_{2}} E_{\mathrm{roll}_{1}}$,

where $E_{\mathrm{roll}_{1}}$ is the rolling energy between monomers with radius $a_{1}, E_{\text {roll }}$ is the rolling energy between monomers with ra$\operatorname{dius} a_{2}$.

As we can see in Fig. 10, the maximum aggregate sizes reached are approximately an order of magnitude higher than in Fig. 7 as it was predicted earlier in this section.

\section{Conclusions and outlook}

We have shown that our representative particle method for aggregation of particles in astrophysical settings works well for standard kernels. It has the usual advantages of Monte Carlo methods that one can add particle properties easily and without loss of computational speed. Moreover, it naturally conserves the number of computational elements, so there is no need to "add" or "remove" particles. Each representative particle represents a fixed portion of the total mass of solids.

Our method may have various possible interesting extensions and applications. Here we speculate on a few of these. For instance, the fact that each representative particle corresponds to a fixed amount of solid mass makes the method ideal for implementation into spatially resolved models such as hydrodynamic simulations of planetary atmospheres or protoplanetary disks. We can then follow the exact motion of each representative particle through the possibly turbulent environment, and thereby automatically treat the stochastic nature and deviation from a Boltzmann distribution of the motion of particles with stopping times of the same order as the turbulent eddy turn-over time. It is necessesary, however, to assure that a sufficiently large number of representative particles is present in each grid cell of the hydrodynamic simulation. For large scale hydrodynamic simulations this may lead to a very large computational demand for the coagulation computation, as well as for tracking the exact motion of these particles. If strong clumping of the particles happens, however, much of the "action" anyway happens in these "clumps", and it may then not be too critical that other grid cells are not sufficiently populated by representative particles. This, however, is something that has to be experimented.
Our representative particle method can in principle also be used to model the sublimation and condensation of dust grains. If a particle sublimates then the representative particle becomes simply an atom or molecule of the vapor of this process. It will then follow the gas motion until the temperature becomes low enough that it can condense again. Other representative particles which are still in the solid phase may represent physical particles that can act as a condensation nucleus. Finally, in our method the properties of the particle can not only change due to collisions, but we can easily implement other environmental factors in the alteration of particle properties.

There are two main drawbacks of the method. First, it only works for large particle numbers, i.e. it cannot treat problems in which individual particles start dominating their immediate environment. Ormel's method and its expected extension do not have this problem. Secondly, the method cannot be accelerated using implicit integration, while Brauer's method can.

All in all we believe that this method may have interesting applications in the field of dust aggregation and droplet coagulation in protoplanetary disks and planetary atmospheres.

Acknowledgements. We wish to thank Frithjof Brauer, Anders Johansen, Patrick Glaschke, Thomas Henning, Jürgen Blum, Carsten Güttler, Carsten Dominik and Dominik Paszun for useful comments. We also thank the anonymous referee for very useful comments that helped to improve the paper and for pointing us to a problem in our presentation of the solution to the particle number conservation paradox.

\section{References}

Blum, J., \& Wurm, G. 2000, Icarus, 143, 138

Brauer, F., Dullemond, C. P., Johansen, A., et al. 2007, A\&A, 469, 1169

Brauer, F., Dullemond, C., \& Henning, T. 2008, A\&A, 480, 859

Dominik, C., Blum, J., Cuzzi, J. N., \& Wurm, G. 2007, in Protostars and Planets V, ed. B. Reipurth, D. Jewitt, \& K. Keil, 783

Dominik, C., \& Tielens, A. 1997, ApJ, 480, 647

Dullemond, C. P., \& Dominik, C. 2005, A\&A, 434, 971

Heim, L.-O., Blum, J., Preuss, M., \& Butt, H.-J. 1999, Phys. Rev. Lett., 83, 3328

Ilgner, M., \& Nelson, R. P. 2006, A\&A, 445, 205

Johansen, A., Klahr, H., \& Henning, T. 2006, ApJ, 636, 1121

Kostinski, A., \& Shaw, R. 2005, Bull. Am. Meteorol. Soc., 86, 235

Mathis, J. S., Rumpl, W., \& Nordsieck, K. H. 1977, ApJ, 217, 425

Mokler, F. 2007, Astron. Nachr., 328, 659

Nakagawa, Y., Nakazawa, K., \& Hayashi, C. 1981, Icarus, 45, 517

Nomura, H., \& Nakagawa, Y. 2006, ApJ, 640, 1099

Ohtsuki, K. O., Nakagawa, Y., \& Nakazawa, K. 1990, Icarus, 83, 205

Ormel, C. W., \& Cuzzi, J. N. 2007, A\&A, 466, 413

Ormel, C. W., Spaans, M., \& Tielens, A. G. G. M. 2007, A\&A, 461, 215

Ossenkopf, V. 1993, A\&A, 280, 617

Paszun, D., \& Dominik, C. 2006, Icarus, 182, 274

Sano, T., Miyama, S. M., Umebayashi, T., \& Nakano, T. 2000, ApJ, 543, 486

Schmitt, W., Henning, T., \& Mucha, R. 1997, A\&A, 325, 569

Semenov, D., Wiebe, D., \& Henning, T. 2004, A\&A, 417, 93

Smoluchowski, M. V. 1916, Z. Phys., 17, 557

Suttner, G., \& Yorke, H. W. 1999, ApJ, 524, 857

Tanaka, H., Himeno, Y., \& Ida, S. 2005, ApJ, 625, 414

Weidenschilling, S. 1984, Icarus, 60, 553

Weidenschilling, S. J. 1997, Icarus, 127, 290

Wetherill, G. W. 1990, Icarus, 88, 336 